\title{
Ecotoxicidade e risco ambiental de fármacos para peixes
}

Ecotoxicity and environmental risk of fish drugs

Ecotoxicidad y riesgo ambiental de los fármacos para los peces

Claudomiro Vinicius Moreno Paschoa

Engenheiro Civil e Biólogo, UNIFEV, Brasil.

viniciuspaschoa@gmail.com

Juliana Heloisa Pinê Américo-Pinheiro Professora Doutora do PPGEC, UNESP - Ilha Solteira/SP, Brasil. 


\section{RESUMO}

Os estudos dos compostos farmacêuticos no ambiente aquáticos estão em evidência. Nos últimos anos tem sido foco de toxicologistas ambientais devido à sua acumulação nestes ecossistemas, dado que a presença de muitas dessas substâncias pode trazer danos irreversíveis a biota. $\mathrm{O}$ objetivo do presente trabalho foi caracterizar e expor os efeitos tóxicos dos fármacos fluoxetina, verapamil, triclosan e fármacos antiepiléticos em espécies de peixes. Muitas drogas projetadas para humanos são susceptíveis de ter efeitos correlatos em vertebrados e invertebrados aquáticos, por isso torna-se relevante o estudo dos fármacos para peixes, visto que a acumulação de produtos farmacêuticos em ambientes aquáticos pode afeta-los diretamente. A ocorrência de fármacos residuais no meio ambiente pode apresentar efeitos adversos em organismos aquáticos e terrestres. $O$ efeito pode ser em qualquer nível da hierarquia biológica, inclusive afetar todo o ecossistema. Com o presente estudo contatou-se que por mais que os estudos do segmento estão em evidencia, ainda assim se faz necessário uma avaliação prudente dos efeitos desses fármacos no meio aquático. Uma vez conhecido os efeitos, se torna viável estabelecer os limites de concentrações para o descarte seguro de efluentes domésticos tratados em corpos receptores.

PALAVRAS-CHAVE: Ambiente aquático. Tratamento de água. Efluente.

\section{ABSTRACT}

Studies of the pharmaceutical compounds in the aquatic environment are in evidence. In recent years it has been the focus of environmental toxicologists due to their accumulation in these ecosystems, since the presence of many of these substances can bring irreversible damage to biota. The objective of the present study was to characterize and expose the toxic effects of fluoxetine, verapamil, triclosan and antiepileptic drugs in fish species. Many drugs designed for humans are likely to have related effects on vertebrates and aquatic invertebrates, so the study of fish drugs is relevant, as the accumulation of pharmaceuticals in aquatic environments can directly affect them. The occurrence of residual drugs in the environment may have adverse effects on aquatic and terrestrial organisms. The effect can be on any level of the biological hierarchy, including affecting the entire ecosystem. With the present study, it was found that although the studies of the segment are in evidence, a prudent evaluation of the effects of these drugs in the aquatic environment is still necessary. Once the effects are known, it is feasible to establish the concentration limits for the safe disposal of treated domestic effluents in receiving bodies.

KEYWORDS: Aquatic environment. Water treatment. Effluent.

\section{RESUMEN}

Los estudios de los compuestos farmacéuticos en el ambiente acuático están en evidencia. En los últimos años ha sido foco de toxicólogos ambientales debido a su acumulación en estos ecosistemas, dado que la presencia de muchas de estas sustancias puede traer daños irreversibles a la biota. El objetivo del presente trabajo fue caracterizar y exponer los efectos tóxicos de los fármacos fluoxetina, verapamilo, triclosan y fármacos antiepilépticos en especies de peces. Muchas drogas diseñadas para humanos son susceptibles de tener efectos correlatos en vertebrados e invertebrados acuáticos, por lo que es relevante el estudio de los fármacos para peces, ya que la acumulación de productos farmacéuticos en ambientes acuáticos puede afectarlos directamente. La aparición de fármacos residuales en el medio ambiente puede tener efectos adversos en organismos acuáticos y terrestres. El efecto puede ser en cualquier nivel de la jerarquía biológica, incluso afectar todo el ecosistema. Con el presente estudio se ha entendido que por más que los estudios del segmento están en evidencia, aun así se hace necesaria una evaluación prudente de los efectos de esos fármacos en el medio acuático. Una vez conocidos los efectos, se hace viable establecer los límites de concentraciones para el descarte seguro de efluentes domésticos tratados en cuerpos receptores.

PALABRAS-CLAVE: Ambiente acuático. Tratamiento de agua. Efluente. 


\section{INTRODUÇÃO}

Os fármacos são compostos utilizados globalmente que tem sido foco da toxicologista ambiental devido à sua acumulação em ecossistemas aquáticos (OGGIER et al., 2010). Ingredientes ativos de compostos farmacológicos são encontrados no ambiente, incluindo águas potáveis e poluídos, sendo que a acumulação de produtos farmacêuticos em ambientes aquáticos pode afetar peixes e outros organismos não alvos (LI et al., 2011).O termo ecotoxicologia pode ser facilmente entendido ao realizar a junção do dos termos: ecologia e toxicidade. A definição de ecologia refere-se ao estudo da interação dos seres vivos entre si e com o meio ambiente em que vivem. Já a toxicologia é uma ciência que procura entender os tipos de efeitos causados por substâncias químicas, bioquímicas e os processos biológicos responsáveis por tais efeitos. O objetivo da ecotoxicologia seria então entender e prever efeitos de substâncias químicas em seres vivos e comunidades naturais (CHAPMAN, 2006).O impacto dos produtos farmacêuticos em organismos aquáticos expostos às concentrações mais baixas muitas vezes é observado após longo período de exposição (THIBAUT; PORTE, 2008). Devido à natureza conservadora da fisiologia dos vertebrados, muitas drogas projetadas para humanos são susceptíveis de ter efeitos semelhantes em vertebrados e invertebrados aquáticos, portanto se torna muito relevante o estudo dos fármacos para peixes, visto que a acumulação de produtos farmacêuticos em ambientes aquáticos pode afetá-los. A principal via de contaminação do meio ambiente com fármacos é o lançamento de esgotos in natura e tratado. Assim, ocorrência de fármacos residuais no meio ambiente pode apresentar efeitos adversos em organismos aquáticos e terrestres (BARRY, 2013).

O efeito pode ser em qualquer nível da hierarquia biológica, inclusive um ecossistema, entretanto, a não existência de programas específicos de monitoramento, dificulta o processo de fiscalização (THIBAUT; PORTE, 2008).

Como apontado, esse trabalho justifica-se pelo aumento indiscriminado do uso de fármacos e seu descarte na maioria das vezes irregulares sendo que a persistência de várias dessas substâncias podem trazer danos irreversíveis à biota, se fazendo necessário o estudo dos efeitos do mesmo nos organismos aquáticos. Assim, o objetivo do presente trabalho foi caracterizar e expor os efeitos tóxicos dos fármacos fluoxetina, verapamil, triclosan e fármacos antiepiléticos em espécies de peixes.

\section{FLUOXETINA}

O cloridrato de fluoxetina é um fármaco antidepressivo utilizado no tratamento de depressão. Assim, o ingrediente ativo da fluoxetina, como de muitos fármacos, não é absorvido completamente pelo corpo humano e chegam às estações de tratamento de esgoto (ETE) e posteriormente aos ambientes aquáticos (HUGGETT et al., 2003). As ETE não apresentam sistemas de tratamento ou mesmo tecnologias adequadas para remoção completa de fármacos e seus resíduos por meio dos efluentes tratados atingem os ecossistemas de água doce. Mesmo em baixas concentrações, esses compostos podem se bioacumular na cadeia trófica e, assim, influenciar o comportamento animal (HUERTA et al., 2016). 
Os estudos que avaliam os efeitos potenciais da fluoxetina nos peixes, em sua maioria, descrevem mudanças no comportamento desses organismos, mesmo as concentrações muito baixas comprovam alterar a velocidade da natação (BARRY, 2013). No peixe lutador Siamês, Betta splendins, concentrações no entorno de $0,54 \mu \mathrm{g} \mathrm{L}^{-1}$ reduzem significativamente sua agressividade (DZIEWECZYNSKI; HEBERT, 2012).

No peixe $B$. splendins, o sucesso reprodutivo masculino é muito dependente da habilidade do macho de competir com outros machos para obter acesso às companheiras e, uma vez seguro, eles também devem defender agressivamente seus ninhos de machos rivais e até mesmo de fêmeas que parecem para atacar e se alimentar de jovens em desenvolvimento (BARRY, 2013). A lateralidade é um fenômeno que se refere ao viés lateral que é induzido pela lateralização cerebral da função cognitiva amplamente estudada em peixes e tem impactos abrangentes tanto na cognição quanto no comportamento geral. Esse fenômeno importante que recebeu muito pouca atenção relacionada aos fármacos no contexto da ecotoxicologia, mas provavelmente é influenciado pela fluoxetina, indica que a fluoxetina atua sobre receptores de serotonina e claramente a função cerebral será fortemente influenciada pela exposição (BISAZZA; DE SANTI, 2003).

Poucos estudos examinaram e relacionaram a lateralidade em função da exposição de fluoxetina. Contudo, estudos de comportamento agressivo em lagartos da anolis sugerem que a fluoxetina pode ter influências assimétricas no cérebro (DECKEL et al., 1998). Assim, há uma boa razão para suspeitar que a fluoxetina irá influenciar a lateralidade em peixes de água doce. Além disso, os comportamentos agressivos nos peixes tendem a ser fortemente lateralizados ao hemisfério esquerdo (BISAZZA; DE SANTI, 2003), de modo que a agressão e a lateralidade tendem a estar intimamente relacionadas em uma ampla gama de vertebrados, incluindo peixes.

Embora poucos estudos tenham determinado qual o impacto dessas mudanças no comportamento da aptidão física, existem alguns sistemas modelo que foram comparativamente bem estudados para que possamos fazer algumas inferências (WONG; CANDOLIN, 2015).

\section{FÁRMACOS ANTIEPILÉPTICOS}

As convulsões espontâneas e recorrentes caracterizam a epilepsia, que é uma das doenças neurológicas mais comuns em todo o mundo (ROBINSON, 2009). Essas crises epilépticas ocorrem devido a um desequilíbrio entre excitação e inibição sináptica que resulta em atividade elétrica hiperescrono de redes neuronais (TINSLEY et al., 2004).

Os fármacos antiepiléticos têm como principal mecanismo de ação a diminuição dos disparos neuronais repetitivos, que por sua vez, atuam no bloqueio de canais iônicos dependentes de voltagem, na potencialização da neurotransmissão gabaérgica (inibitória) e na diminuição da neurotransmissão excitatória glutamaérgica (HUMMEL et al., 2006).Níveis aumentados podem alterar o funcionamento neurológico e sua síntese ou liberação excessiva está fortemente relacionada à crise convulsiva (TINSLEY et al., 2004). 
O peixe zebra (Danio rerio) é um pequeno teleósteo que surgiu como um importante modelo experimental. Vários sistemas de neurotransmissão já foram identificados neste peixe e seu genoma é muito similar ao de mamíferos incluindo a espécie humana (BARABAN et al., 2005). O genoma deste peixe é bem caracterizado e compartilha muitas semelhanças com o genoma humano (BISAZZA; DE SANTI, 2003). Estudos demonstraram que as larvas de peixe zebra e os adultos poderiam ser modelos potenciais para estudar convulsões (HUMMEL et al., 2006).

As larvas do peixe expostas à pentilenotetrazol (PTZ), um agente convulsivo, apresentaram alterações estereotipadas comportamentais, eletrográficas e moleculares, semelhantes às alterações relatadas nos modelos de convulsões de roedores (BISAZZA; DE SANTI, 2003). Além disso, a exposição à PTZ, cafeína e picrotoxina em $D$. rerio adulto também leva a respostas comportamentais semelhantes às crises. Conforme relatado em modelos de roedores, convulsões induzidas quimicamente no peixe zebra podem ser suprimidas por drogas antiepilépticas clássicas, como valproato, diazepam e carbamazepina (HUMMEL et al., 2006).

\section{VERAPAMIL}

O verapamil é um fármaco do grupo dos antiarrítmicos que pode ser usado no tratamento de transtornos cardiovasculares. Esse composto prolonga o tempo de condução de cada impulso cardíaco diminuindo a frequência cardíaca e está na lista da organização mundial da saúde, entre os medicamentos essenciais em um serviço de saúde. Em peixes pode induzir o estresse oxidativo e afetar a estrutura molecular no cérebro e músculo do animal. As espécies reativas de oxigênio em excesso reagem com macro moléculas biológicas para elevar o nível de peroxidação lipídica e alterar as atividades das enzimas antioxidantes, bem como a desnaturação de proteínas em um sistema (NWANI et al., 2015).

Overturf et al. (2011) constataram que após a exposição a uma concentração de $600 \mu \mathrm{g} \mathrm{L}{ }^{-1}$ de verapamil, larvas do peixe Pimephales promelas apresentaram diminuição significativa na taxa de crescimento. Além disso, uma exposição de 96 h da truta arco-íris (Oncorhynchus mykiss) ao verapamil a uma concentração de $27 \mathrm{\mu g} \mathrm{L}^{-1}$ afeta o sistema de resposta antioxidante e também resulta em mudanças fisiológicas e bioquímicas após 42 dias do período de exposição.

Nallani et al. (2016) encontraram alto nível de acumulação de verapamil nos tecidos do peixe Leporinus friderici e do peixe-gato (Ictalurus melas) na concentração de $500 \mu \mathrm{g} \mathrm{L}^{-1}$ do medicamento após 28 dias de exposição.

A exposição prolongada de tilápia (Oreochromis niloticus) ao verapamil afeta especialmente a atividade da acetilcolinesterase e das enzimas relacionadas ao estresse oxidativo, bem como o gene relacionado ao estresse, apontando que a acetilcolinesterase desempenha um papel ativo na regulação da atividade elétrica neuronal na liberação de neurotransmissor e na plasticidade sintética no sistema nervoso central (LIU et al., 2013). As proteínas de choque térmico são uma família de proteínas altamente conservada e omnipresente que responde a uma ampla variedade de estresse físico e metabólico, incluindo estresse oxidativo e térmico (LIU et al., 2013).

\section{TRICLOSAN}


O triclosan é um fármaco antimicrobiano, pertencente ao grupo dos fenoxifenois policlorados. É usado como antisséptico (efetivo contra bactérias gram negativas, bem como gram positivas) e também como conservante em cosméticos. É encontrado principalmente em medicamentos, sabonetes, loções, desodorantes e cremes dentais, e pode atingir o ambiente aquático por meio de efluentes não tratados (fezes, urina, após o uso do consumidor) (PEREZ 2013) ou por efluentes tratados por sistemas que não removem essa substância no processo de tratamento (BOCK et al., 2010).

Embora as avaliações de risco tenham encontrado um risco ecológico potencial mínimo devido à exposição ao triclosan em sistemas aquáticos, muita atenção tem sido focada nos potenciais efeitos na saúde humana associados aos riscos de exposições ambientais ao triclosan (CAPDEVIELLE et al., 2008)

Esse antimicrobiano afeta as fibras musculares esqueléticas e as células cardíacas o que pode colocar em risco a sobrevivência de peixes (BOCK et al., 2010). Segundo Carpdevielle et al. (2008), a presença de triclosan em águas superficiais e sedimentos podem impedir contrações musculares e diminuir a velocidade de natação de peixes.

\section{CONSIDERAÇÕES FINAIS}

Os fármacos e os seus metabolitos são relatados como causadores de efeitos ecotoxicológicos em peixes, visto que são detectados em ecossistemas aquáticos. Atualmente, a preocupação com a presença de fármacos em ambientes aquáticos e seus possíveis impactos ambientais é crescente.

A literatura mostra que muitos desses fármacos residuais foram detectados em águas superficiais e em efluentes de ETE. Pouco é conhecido sobre o efeito dessas substâncias no meio ambiente. Outro ponto pouco explorado refere-se a uma avaliação de impacto no meio ambiente por meio de avaliações ecotoxicológicas.

Uma avaliação criteriosa dos efeitos desses fármacos no meio aquático é indispensável. Uma vez conhecido os efeitos, será necessário estabelecer limites de concentrações para o descarte seguro de efluentes tratados em corpos receptores.

O monitoramento da eficiência de remoção desses fármacos pelos processos convencionais de tratamento de efluentes domésticos das ETE é de grande importância, no futuro, podem ser necessárias adaptações, ou mesmo implantar outros processos de tratamento que complementem a remoção adequada desses fármacos.

\section{REFERÊNCIAS BIBLIOGRÁFICAS}

BARABARAN, S.C. et al. Pentylenetetrazole induced changes in zebrafish behavior, neural activity e c-fos expression. Neuroscience. v. 131, p. 759-768, 2005.

BARRY, M. J. Effects of fluoxetine on the swimming and behavioural responses of the Arabian killifish. Ecotoxicology, n.22, p. 425-432, 2013. 
BISAZZA, A.; DE SANTI, A. Lateralization of aggression in fish. Behav. Brain Research Bulletin, n.141, p. 131-136, 2003.

BOCK, M. et al. Probabilistic application of a fugacity model to predict triclosan fate during wastewater treatment. Integr. Environ. Integrated Environmental Assessment and Management, v.6, n.3, p. 340-393, 2010.

CAPDEVIELLE, M. et al. Consideration of exposure and species sensitivity of triclosan in the freshwater environment. Integr. Environ. Integrated Environmental Assessment and Management, v.4, n.1, p.15-23, 2008.

CHAPMAN, P. M. Emerging substances - Emerging problems? Environmental Toxicology and Chemistry, v.25, n.6, p.1445-1447. 2006.

DECKEL, A. W. et al. Lateralized effects of ethanol on aggression and serotonergic systems. Anolis carolinensis. Brain Research Bulletin, n.807, p.38-46, 1998.

DZIEWECZYNSKI, T. L.; HEBERT, O. L. Fluoxetine alters behavioral consistency of aggression and courtship in male Siamese fighting fish, Betta splendens. Physiology Behavior Journal, n.107, p.92-97, 2012.

HUERTA, B. et al. Anti-anxiety drugs and fish behavior: establishing the link between internal concentrations of oxazepam and behavioral effects. Environmental Toxicology Chemistry, n.35, p.2782-2790, 2016.

HUGGETT, D.B. et al. A theoretical model for utilizing mammalian pharmacology and safety data to prioritize potential impacts of human pharmaceuticals to fish. Human and Ecological Risk Assessment, n.9, p.1789-1799, 2003.

HUMMEL, D. et al. Simultaneous determination of psycho-active drugs and their metabolites in aqueous matrices by liquid chromatography mass spectrometry. Environmental Science and Technology, v.40, p.7321-7328, 2006.

LI, Z. H. et al. Chronic toxicity of verapamil on juvenile rainbow trout (Oncorhynchus mykiss): effects on morphological indices, hematological parameters and antioxidant responses. Journal of Hazardous Materials, n.185, p.870-880, 2011.

LIU, C. M. et al. Protective effect of puerarin on lead-induced mouse cognitive impairment via altering activities of acetyl cholinester-ase, monoamine oxidase and nitric oxide synthase. Journal of Toxicology and Pharmacology, n.35, p.502-510, 2013.

NALLANI, G.C. et al. Bioconcentration of two basic pharmaceuticals, verapamil and clozapine, in fish. En-viron. Environmental Toxicology Chemistry, n.35, p. 593-603, 2016.

NWANI, C. D. et al. Oxidative stress and biochemical responses in the tissues of African catfish, Clarias gariepinus juvenile following exposure to primextra herbicide. Drug and Chemical Toxicology, n.38, p.278-285, 2015.

OGGIER, D. M. et al. Effects of diazepam on gene expression and link to physiological effects in different life stages in zebrafish Danio rerio. Environmental Science and Technology, n.44, p.7685-7691, 2010.

OVERTURF, M.D. et al. Early life stage toxicity of eight pharmaceuticals to the Fathead Minnow, Pimephales promelas. Archives of Environmental Contamination and Toxicology, n.62, p.455-464, 2011.

PEREZ, A. L. et al. Triclosan occurrence in freshwater systems in the United Sates (1999-2012): a meta-analysis. Environ. Environmental Toxicology Chemistry, n.32, p. 1479-1487, 2013.

ROBINSON, P. D. Behavioural toxicity of organic chemical contaminants in fish: appli-cation to ecological risk assessments (ERAs). Canadian Journal of Fisheries and Aquatic Sciences, n.66, p. 1179-1188, 2009.

THIBAUT, R.; PORTE, C. Effects of fibrates, anti-inflammatory drugs and antidepres-sants in the fish hepatoma cell line PLHC-1: cytotoxicity and interactions with cyto-chrome P450 1A. Toxicology in Vitro, n.22, p.1128-1135, 2008. 
TINSLEY, M.R., QUINN, J.J., FANSELOW, M.S. The role of muscarinic and nicotinic cholinergic neurotransmission in aversive conditioning: comparing pavlovian fear conditioning and inhibitory avoidance. Learning \& Memory. v. 11, p. 35-42, 2004.

WONG, B. B.; CANDOLIN, U. Behavioral responses to changing environments. Behavioral Ecology, v.26, p. 665-673, 2015. 